\title{
L'HABITAT : ÊTRE AU BON ENDROIT AU BON MOMENT ?
}

\author{
C. LÉVÊQUE \\ ORSTOM, 213 rue La Fayette, \\ 75480 Paris Cedex 10, France.
}

\section{RÉSUMÉ}

La variabilité spatiale et temporelle est une des caractéristiques des milieux lotiques en raison notamment des fluctuations du débit et du niveau de l'eau. Dans cet environnement, le poisson recherche en permanence un compromis entre la variabilité du milieu et l'accomplissement de ses besoins vitaux comme la nécessité de se reproduire, de se protéger des prédateurs, et de s'alimenter à différents stades de son développement. Son habitat (par définition, le milieu géographique propre à la vie d'une espèce animale ou végétale) résulte de cette recherche de compromis.

L'héritage phylogénique fait peser sur l'espèce un certain nombre de contraintes abiotiques, biologiques et comportementales qui fixent le cadre de ses besoins en terme d'habitat. Mais les individus développent également des tactiques qui sont des réponses adaptatives aux modifications du milieu, et qui peuvent se traduire par des comportements alternatifs. Certains résultats font également penser qu'il y a possibilité d'apprentissage et de choix.

L'habitat est donc une notion essentiellement dynamique, une référence spatiale et temporelle : la position qu'occupe à un instant donné un individu parvenu à un certain stade de développement, cherchant à optimiser le nécessaire compromis entre différentes contraintes biologiques et écologiques, dans un milieu lui-même très variable.

Dans ce contexte, l'habitat ne peut être défini seulement par les caractéristiques physiques du milieu, comme ce fut souvent le cas jusqu'ici. Il faut rechercher une typologie qui tienne compte des comportements biologiques et quatre grands types d'organisation spatio-temporelle sont proposés :

- la zone de stabulation ou zone de repos pour l'individu qui cherche avant tout un abri temporaire vis-à-vis du milieu ou de prédateurs,

- la zone d'activité qui est l'échelle de référence pour les cycles nycthéméraux et lunaires correspondant notamment à la recherche de nourriture,

- la niche ontogénique qui correspond à l'ensemble des milieux dont une espèce a besoin pour accomplir son cycle biologique. Cette échelle qui inclut les déplacements nécessaires à la reproduction et au développement, est d'étendue variable selon que les poissons auront un comportement de type sédentaire ou nomade,

- l'échelle de la métapopulation qui correspond aux différents bassins hydrographiques dans lesquels l'espèce est présente.

\section{HABITAT : TO BE AT THE RIGHT PLACE AT THE RIGHT TIME?}

\section{SUMMARY}

Spatial and temporal variability, as a result of temporal changes in discharge pattern and water levels, is a characteristic of river systems. Fish permanently seeks a trade-off between habitat variability and the need to accomplish vital functions such as reproduction, escaping predation, finding adequate food according to the stage of development. Fish habitat (defined here as the geographical range of an animal or plant species) is the result of this trade-off. 
Phylogenetic heritage imposes several abiotic, biological and behavioural constraints that determine habitat needs. However, individuals develop tactics that are adaptation to environmental changes and may result in alternative behaviour. A few results also seem to indicate some possibility of choice and learning.

Fish habitat is therefore a dynamic concept with spatial and temporal implications, it is the position occupied at a given time by a fish reaching a given developmental stage and searching for the best trade-off between its biological and ecological constraints in a highly variable freshwater environment.

In this context, habitat cannot be defined only on physical criteria, as was often the case so far. There is a need to take into account biological behaviour, and a general typology based on four main types of combined temporal and spatial scales may be proposed :

- the resting area or refuge zone of an individual fish sheltering from predators or abiotic environmental factors ;

- home range, in which biological and behavioural events (including foraging activity) are rhythmically driven by diel and lunar cycles;

- ontogenetic niche, which includes the habitats used by the different developmental stages. Its spatial limits include the range of spawning migrations and juvenile habitats. Its extent therefore depends on the sedentary or nomadic behaviour of the species involved;

- metapopulation range, which covers the patchy biogeographical distribution of the species, eventually over isolated catchments.

\section{L'HABITAT OU L'ART DES COMPROMIS}

Dans les systèmes aquatiques, les différentes espèces de poissons ne se sont pas distribuées au hasard et l'une des questions qui suscite l'intérêt des écologistes est la suivante : comment peut-on expliquer qu'un poisson se situe dans un lieu donné à un moment donné qui est le plus souvent celui de l'observation ? Parmi les nombreuses raisons possibles, il faut considérer qu'un poisson, au cours de chacune des étapes de son développement, doit faire face à trois contraintes essentielles :

- se protéger des contraintes du milieu (courant, oxygène, température, etc...) ainsi que des prédateurs et éventuellement des compétiteurs de manière à assurer sa survie,

- se nourrir dans les meilleures conditions pour assurer sa croissance et sa survie individuelle sachant que ses exigences alimentaires varient au cours de son développement,

- à l'état adulte, se reproduire dans les conditions les plus favorables de manière à assurer la survie de l'espèce. Il est ainsi amené à se déplacer (migrations) et à développer éventuellement des formes de protection des oeufs ou des alevins (soins parentaux).

Une deuxième question vient immédiatement à l'esprit : comment l'utilisation de l'espace évolue-t-elle dans le temps ? En effet, la variabilité spatiale et temporelle est une des caractéristiques des milieux lotiques en raison notamment des fluctuations de débit et de niveau de l'eau qui modifient considerablement la nature et l'étendue du milieu habitable. II y a également des rythmes nycthéméraux synchronisés par des stimulus comme la lumière ou la température, et des rythmes saisonniers qui concernent différents facteurs du milieu dont la température et l'hydrodynamique. Il existe en outre une certaine variabilité interannuelle, aucune année n'étant strictement comparable à la précédente, et des événements exceptionnels sont également susceptibles d'affecter épisodiquement les milieux aquatiques.

Tout endroit donné dans un hydrosystème est donc soumis à d'importantes fluctuations. Dans cet environnement très variable, le poisson va rechercher en permanence des compromis de manière à optimiser ces trois fonctions principales : 
s'alimenter, se reproduire, et s'abriter des prédateurs. Et son habitat (par définition, le milieu géographique propre à la vie d'une espèce animale ou végétale) résultera de cette recherche de compromis entre la variabilité du milieu et l'optimisation de ses besoins vitaux. Au cours de sa vie, et en fonction de son activité journalière ou saisonnière, une même espèce pourra ainsi occuper successivement plusieurs types d'habitats.

A titre d'exemple, les compromis peuvent être de quitter l'abri pour se nourrir en s'exposant aux prédateurs, ou de migrer pour se reproduire dans des zones pauvres en ressources alimentaires mais favorables au développement des oeufs et des larves. Un problème majeur pour tout poisson est de savoir comment partager son temps et utiliser son énergie pour se nourrir et/ou pour se reproduire de manière à ce que le succès de la reproduction soit maximal. En minimisant l'énergie nécessaire à la recherche et à la capture des proies, le poisson aura plus d'énergie à consacrer à la croissance et au métabolisme (HART, 1986). Face aux différentes options qui leurs sont offertes dans le milieu naturel, les animaux ne choisissent pas au hasard mais au contraire accomplissent les activités qui assurent un succès reproducteur plus élevé (PULLIAM, 1989). Cette recherche de compromis entre des exigences biologiques aurait pour objectif d'optimiser ce qu'il est convenu d'appeler le "fitness", c'est-à-dire la contribution relative d'un individu aux futures générations.

\section{SE NOURRIR ET CROITTRE}

Au cours de leur existence la taille et le poids des poissons changent considérablement. L'ontogenèse est le processus qui correspond à la différenciation des stades de développement au cours de la vie de l'organisme. Chez les poissons, on distingue généralement différentes périodes (BALON, 1985, 1990) :

- la période embryonnaire qui commence avec la fertilisation et se caractérise par une nutrition exclusivement endogène à partir du vitellus de l'oeuf,

- la période larvaire débute avec le passage graduel mais rapide d'une alimentation endogène à une alimentation exogène. Cette période larvaire se caractérise par la présence d'organes larvaires temporaires,

- la période juvénile débute lorsque les nageoires sont bien différenciées et que tous les organes temporaires ont été remplacés par des organes définitifs. Elle se termine avec la maturation des premiers gamètes. C'est généralement une période de croissance rapide parfois caractérisée par une coloration spécifique,

- la période adulte débute avec la maturation des premiers gamètes. Elle se caractérise par une diminution du taux de croissance somatique,

- une période de sénescence est parfois distinguée.

Ces différentes phases du développement correspondent à des exigences écologiques, physiologiques, et biologiques différentes, ce qui suppose dans beaucoup de cas la fréquentation de biotopes différents.

\section{- Changements de régime alimentaire au cours du développement}

Les besoins nutritionnels et le comportement alimentaire des poissons se modifient au cours des phases du développement (LAUZANNE, 1975 ; ROSS, 1986 ; WINEMILLER, 1989). Les juvéniles de petite taille, peu vagiles, ne peuvent exploiter que des particules de petite taille également : phyto ou zooplancton. Avec l'augmentation de la taille du poisson et de ses capacités de déplacement, la taille et la nature des proies évoluent. Chez les poissons ichtyophages à l'état adulte, des changements morphologiques associés à une plus grande spécialisation s'accompagnent de changements dans la taille et la nature des proies.

Quelle conséquence en tirer sur le plan de l'habitat? Les proies ne sont pas distribuées au hasard dans les milieux aquatiques, et leur disponibilité peut varier au cours de l'année. Dans les milieux fluviaux par exemple, les proies planctoniques sont essentiellement abondantes dans les milieux calmes, c'est-à-dire dans les annexes fluviales où les juvéniles de beaucoup d'espèces vont trouver les conditions qui leurs sont 
favorables tant sur le plan de la nutrition que sur celui des conditions physiques de l'habitat. L'existence et l'étendue de ces milieux sont étroitement dépendantes de l'hydrologie et notamment du niveau de l'eau. Il faut donc qu'il y ait bonne synchronisation entre d'une part l'apparition des larves et d'autre part l'existence de proies favorables dans l'habitat correspondant (hypothèse du "match-mismatch» de CUSHING, 1982).

\section{- Stratégies de recherche et de partage de la nourriture}

Les stratégies alimentaires peuvent être vues comme des systèmes de prise de décision pour répondre à des questions du type : où un individu doit-il se nourrir, ou vers quelle proie doit-il diriger sa recherche ? (CEZILLY et al., 1991). Le poisson doit ainsi décider du moment où il va se nourrir, du lieu et de la durée de la période de nutrition, des proies qui sont les plus adéquates (taille et valeur nutritionnelle), de la manière dont il va rechercher ces proies et les capturer. II s'agit de faire face efficacement à différentes contraintes de l'environnement comme la compétition, la raréfaction de la nourriture, les variations imprévisibles de la ressource.

Un axiome fondamental est que les stratégies alimentaires ont été façonnées au cours de la sélection naturelle, et que toute décision tend à optimiser certaines variables comme le taux d'assimilation de l'énergie qui est en dernier ressort corrélé au concept de "fitness" (PYKE, 1984). Mais un certain nombre de résultats font penser que les poissons ont également la possibilité d'apprendre et de pratiquer des comportements alternatifs qui leurs permettent d'être plus efficaces dans la recherche des proies et de vivre plus longtemps (HART, 1986).

\section{disponibles}

- Spécialisation trophique et radiation adaptative : utiliser au mieux les ressources

L'occupation de l'ensemble de l'espace aquatique disponible par des populations d'une même espèce de poisson considérée comme l'ancêtre commun favorise la spéciation car les colonisateurs développent des adaptations morphologiques et comportementales spécifiques à leur nouveau mode de vie. On désigne par radiation adaptative le phénomène de spéciation résultant de la colonisation de plusieurs niches d'un même système écologique par une population ancestrale. On utilise également le terme "foule d'espèces" pour désigner des groupes monophylétiques d'espèces très proches issues d'un ancêtre commun et coexistant dans un même milieu (GREENWOOD, 1984), dont les spécialisations trophiques sont associées à des comportements et des exigences écologiques différentes.

Un exemple fameux de radiation adaptative chez les poissons est celui des Cichlidés des grands lacs d'Afrique de l'Est. Ainsi, les Cichlidés du lac Victoria paraissent descendre d'une seule espèce d'origine fluviale qui aurait colonisé le lac et donné naissance aux quelque 300 espèces vivantes qui occupent toutes les grandes niches écologiques disponibles pour les poissons d'eau douce.

La spécialisation du comportement alimentaire est probablement un élément important du succès de la radiation adaptative. Cette spécialisation a sans aucun doute contribué à la sténotopie, à la philopatrie, et à une spécialisation du comportement reproducteur aboutissant à la spéciation (RIBBINK, 1990). Mais beaucoup d'espèces ont néanmoins conservé la possibilité d'utiliser différentes ressources alimentaires, et de changer de tactique de manière opportuniste.

\section{SE REPRODUIRE : HÉRITAGE ET ADAPTABILITÉ}

La stratégie de reproduction d'une espèce de poisson dans un environnement donné, est un ensemble de traits biologiques comme l'âge à la première reproduction, la relation taille (ou âge) et fécondité, le comportement reproductif, la saison de reproduction, la taille des gamètes, etc.. Mais un individu peut également développer des tactiques, qui sont en réalité des variations par rapport au schéma de reproduction typique, de manière à répondre avec succès à des modifications des facteurs de l'environnement. II s'agit alors d'un comportement adaptatif à des conditions écologiques particulières qui a pour objectif d'assurer la survie de l'espèce. 
BALON $(1985,1990)$ distingue deux grands types de trajectoires ontogéniques dans les modèles de cycle biologique.

Dans le modèle indirect, les oeufs sont généralement petits et produits en grand nombre. Ils donnent naissance à des jeunes larves incomplètement développées, de petite taille, avec seulement un faible volume de vitellus qui est insuffisant pour produire le phénotype définitif. Ces jeunes larves doivent donc se nourrir sur des particules de petite taille pour achever leur développement et sont très vulnérables durant cette période.

Dans le modèle de développement direct, les poissons produisent au contraire un nombre restreint d'oeufs de grande taille avec une grande quantité de vitellus qui permet le développement de l'embryon jusqu'à un stade avancé. La période larvaire est ainsi réduite ou supprimée, et les juvéniles déjà bien formés, et donc moins vulnérables, lorsqu'ils entament leur recherche de nourriture exogène. Labeotropheus, un Cichlidé incubateur buccal du lac Malawi, est un bon exemple de ce type de poisson qui libère un juvénile de grande taille $(14 \%$ de celle de l'adulte), 31 jours seulement après la fertilisation (BALON, 1977).

On a donc affaire ici à deux grands types de stratégies : le développement indirect consiste à produire un grand nombre d'oeufs qui seront soumis à une forte mortalité, mais qui libéreront les parents pour d'autres activités dès la ponte, alors que le développement direct consiste à investir dans la survie d'un faible nombre d'individus, ce qui demande aux parents un investissement énergétique prolongé. Dans un cas (développement direct) les poissons auront un comportement de type sédentaire, éventuellement territorial. Dans l'autre cas (développement indirect) on aura affaire à des espèces nomades susceptibles de couvrir de grandes distances y compris pour se reproduire. Ces deux grandes stratégies ont des conséquences sur le comportement des poissons en terme d'occupation de l'espace.

\section{- Guildes de reproduction}

Il y a eu différents essais pour élaborer une classification générale des modes ou styles de reproduction des poissons. Celle proposée par BALON (1990), basée sur le degré de soins parentaux (éthologie) et les sites de reproduction (écologie), est généralement acceptée. Cet auteur retient trois grands ensembles éthologiques quelle que soit l'origine phylétique des espèces, à l'intérieur desquels on distingue des sousensembles étho-écologiques (tableau I):

Tableau I : Les trois grands ensembles éthologiques concernant le comportement reproducteur et les caractéristiques biologiques qui leurs sont associées (d'après BALON, 1985).

Table I : The three ethological sections of reproductive guilds and their associated biological characteristics (from BALON, 1985).

\begin{tabular}{|c|c|c|}
\hline Non gardien & Gardien & Porteur \\
\hline $\begin{array}{l}\text { pondeurs de pleine eau } \\
\text { ou dans des caches }\end{array}$ & $\begin{array}{l}\text { choix de substrats de ponte } \\
\text { construction de nids }\end{array}$ & $\begin{array}{l}\text { incubation interne } \\
\text { ou externe }\end{array}$ \\
\hline pas de soins parentaux & soins parentaux & soins parentaux \\
\hline forte fécondité & faible fécondité & faible fécondité \\
\hline développement indirect & développement direct & développement indirect \\
\hline $\begin{array}{l}\text { investissement à faible } \\
\text { coût sur un grand nombre }\end{array}$ & $\begin{array}{l}\text { investissement à coût élevé } \\
\text { sur un petit nombre }\end{array}$ & $\begin{array}{l}\text { investissement à coût élevé } \\
\text { sur un petit nombre }\end{array}$ \\
\hline
\end{tabular}


1 - les poissons qui ne s'occupent pas de leurs oeufs une fois qu'ils ont été pondus, parmi lesquels on peut distinguer ceux qui les déposent simplement sur des substrats ouverts ou qui les cachent,

2 - les poissons qui gardent leurs oeufs soit dans des nids, soit au niveau de substrats préalablement sélectionnés ou aménagés,

3 - les poissons qui transportent leurs oeufs durant au moins une portion de la période embryonnaire. Chez les porteurs externes, les oeufs sont portés à la surface du corps ou dans la bouche, ou dans des structures s'ouvrant sur l'extérieur. Chez les porteurs internes, les oeufs sont fécondés et transportés dans le corps.

Chaque sous-ensemble étho-écologique comprend différentes guildes de reproduction basées sur la combinaison de critères morphologiques, comportementaux, et écologiques.

La définition de l'habitat d'une espèce doit forcément tenir compte du comportement reproducteur de cette espèce et de la nécessité de pondre dans les meilleures conditions. En particulier, la ponte a lieu généralement à une période où les conditions environnementales sont en principe les plus favorables à la survie des oeuts et des larves, et beaucoup d'espèces ont un cycle saisonnier de reproduction. Dans les grands fleuves tropicaux, le régime hydrologique, ou plus exactement l'ensemble des conditions climatiques qui prévalent au début de la crue, paraît constituer le principal régulateur de la reproduction. Pour beaucoup d'espèces la ponte coïncide avec la crue et peut avoir lieu du début de la crue jusqu'à son maximum, mais plus rarement durant la décrue.

\section{- Soins parentaux et comportements territoriaux}

On appelle soins parentaux toute aide qui est apportée par les parents et qui a pour but d'assurer une meilleure survie de l'oeuf après sa formation. Cette aide peut aller de la construction de nids jusqu'à la garde des oeufs et des alevins. La pratique de soins parentaux est assez répandue chez les poissons et notamment dans la famille des Cichlidés (KEENLEYSIDE, 1991). Leur fonction principale est de protéger les jeunes contre les prédateurs.

La pratique de soins parentaux peut s'accompagner de comportements territoriaux liés à la compétition et à la défense d'un territoire de reproduction. Le territoire dans ce cas devient une ressource indispensable pour pondre, ressource dont la disponibilité est limitée.

L'incubation buccale est plus spécialisée et d'origine plus récente. L'avantage adaptatif est de mettre les embryons à l'abri des prédateurs et de limiter la compétition pour les sites de reproduction. On peut y voir également un moyen de se libérer de la dépendance d'un substrat pour se reproduire, quand l'espace benthique disponible est très recherché.

On a suggéré que la pratique des soins parentaux s'était développée chez les poissons occupant des milieux caractérisés par leur imprédictibilité spatiale et temporelle (WOOTTON, 1990), afin de limiter les dangers inhérents à cette imprédictibilité. Cette théorie mériterait cependant d'être vérifiée par des observations.

\section{SE PROTÉGER}

Les changements d'habitat au cours du développement sont liés à deux grands types de comportement: d'une part utiliser des habitats qui soient compatibles avec les performances du poisson en termes de capacités physiques et physiologiques, et d'autre part limiter les risques de prédation.

SEMPESKI et GAUDIN (1995) ont mis en évidence la dynamique de l'utilisation de l'habitat chez les jeunes ombres (Thymallus thymallus) au cours de l'ontogenèse, et en fonction du rythme nycthéméral. Avec l'augmentation de la taille, les jeunes ombres d'abord cantonnés près des berges dans les eaux calmes, gagnent progressivement le chenal durant la journée. La nuit par contre, la plupart des juvéniles se cantonnent dans les 
eaux mortes. La distribution verticale varie également : le jour les alevins se nourrissent dans la colonne d'eau, alors que la nuit les poissons sont presque tous sur le fond.

Un moyen de se protéger des prédateurs est d'occuper un habitat où ces derniers n'ont pas la possibilité d'exercer une pression efficace. On a souvent mentionné que les zones inondées encombrées de végétation offraient de bons abris aux juvéniles d'espèces qui se reproduisaient lors de la période de crue.

\section{CONCLUSIONS}

L'habitat est une notion essentiellement dynamique. C'est une référence spatiale et temporelle : la position qu'occupe à un instant donné, dans un milieu très variable, un individu parvenu à un certain stade de développement et qui cherche à optimiser les compromis qu'il doit assurer entre différentes contraintes biologiques et écologiques.

\section{adaptatives \\ - L'habitat : conséquence d'un héritage phylogénique et de stratégies}

Le lieu dans lequel vit une espèce, c'est-à-dire son environnement physique, chimique et biologique, est le résultat de compromis entre différentes contraintes qui, pour une bonne part, sont liées à l'héritage de traits vitaux sélectionnés par l'évolution. C'est le cas, par exemple, pour les comportements reproducteurs, pour les exigences écologiques ou physiologiques, pour les habitudes et spécialisations alimentaires. L'héritage phylogénique fait donc peser sur l'espèce un certain nombre de contraintes abiotiques, biologiques et comportementales qui vont déterminer ses besoins en terme d'habitat.

La variabilité du génome permet néanmoins aux espèces de développer des tactiques, qui sont des réponses adaptatives aux modifications du milieu dans lequel vit le poisson. Cette plasticité peut être vitale pour la survie de l'espèce qui, selon les conditions ambiantes qui lui sont offertes et auxquelles elle ne peut échapper, peut développer des comportements alternatifs.

Enfin, les recherches commencent à mettre en évidence la possibilité d'un apprentissage, ce qui aurait pour conséquence d'élargir encore la possibilité pour une espèce d'occuper de nouveaux milieux.

Les conséquences en terme d'habitat sont importantes (Fig. 1). Si l'héritage phylogénétique contraint le poisson à fréquenter un type de milieu, la variabilité génétique permettra à certains individus d'étendre la gamme des limites que l'espèce peut supporter. La sélection naturelle peut conduire à favoriser ces génotypes et donc à modifier le comportement de l'espèce vis-à-vis de l'habitat.

\section{- La niche ontogénique et ses implications}

L'espèce, lorsque différents stades de développement sont présents simultanément, occupe également des espaces différents. II est important de souligner que le cycle biologique ne peut s'accomplir que si l'individu trouve les conditions nécessaires à son développement à chacune des étapes de l'ontogenèse (du développement). La niche ontogénique est ainsi l'ensemble des habitats et des ressources qui sont nécessaires au bon déroulement de ce cycle biologique.

Ėtre au bon moment au bon endroit signifie donc qu'il doit y avoir une excellente synchronisation entre l'ontogenèse et les changements du milieu. On peut illustrer ces propos par le schéma synthétique proposé par LOWE-McCONNELL (1985) sur le cycle des événements et le cycle biologique des poissons dans les grands fleuves tropicaux ayant des zones d'inondation (Fig. 2).

Une des conclusions pratiques de la notion de niche ontogénique pour la gestion des espèces et des espaces aquatiques, est qu'il faut prendre en considération tous les milieux dont l'espèce peut avoir besoin au cours de son développement. II ne suffit pas de préserver les biotopes indispensables aux adultes pour assurer la pérennité d'une espèce, il faut également s'assurer qu'elle pourra trouver les conditions favorables à la reproduction et à la croissance des larves. 


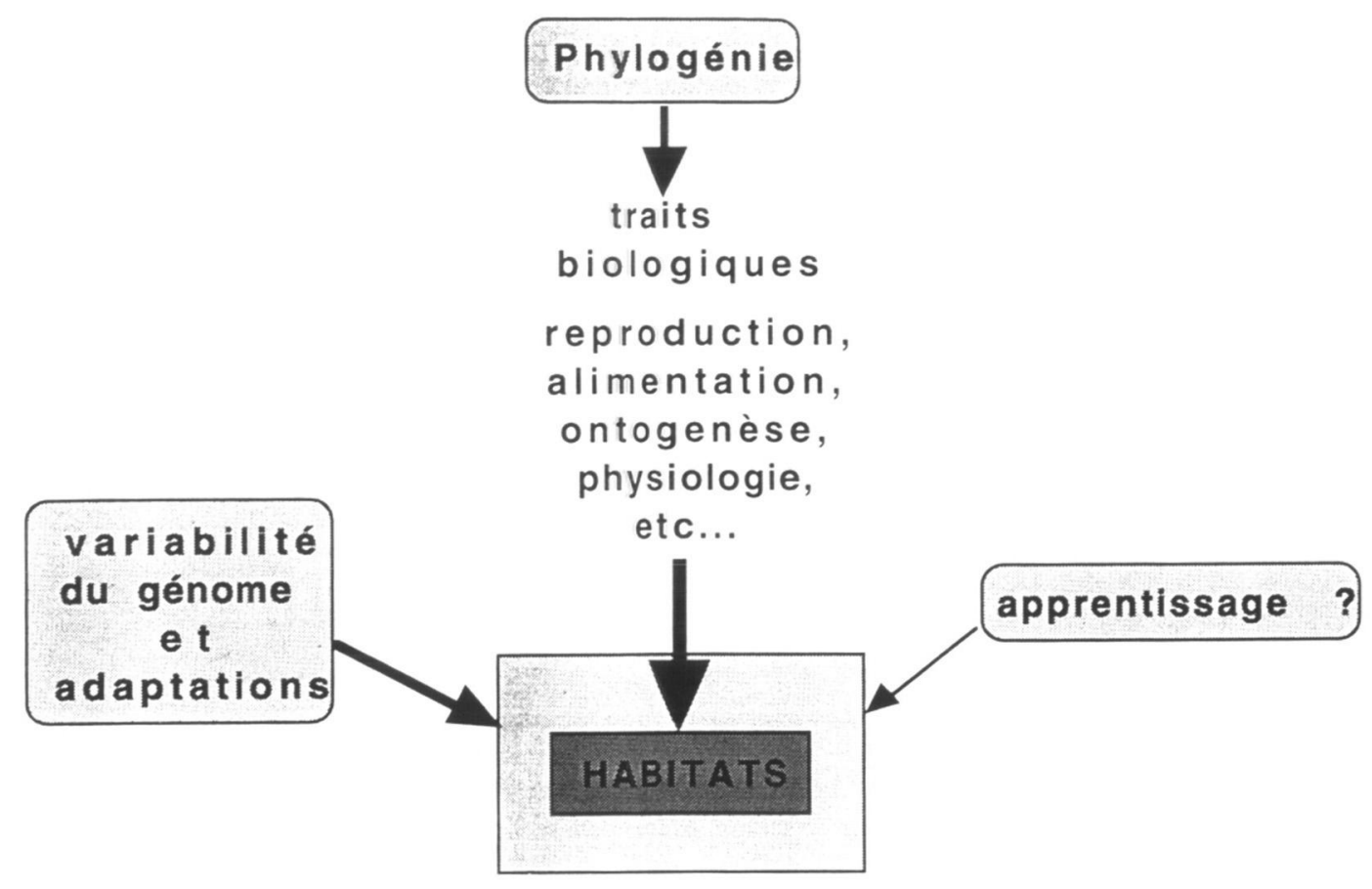

Figure 1 : Principaux facteurs responsables de la sélection de l'habitat par les poissons. Figure 1 : Main factors involved in habitat selection by fish.

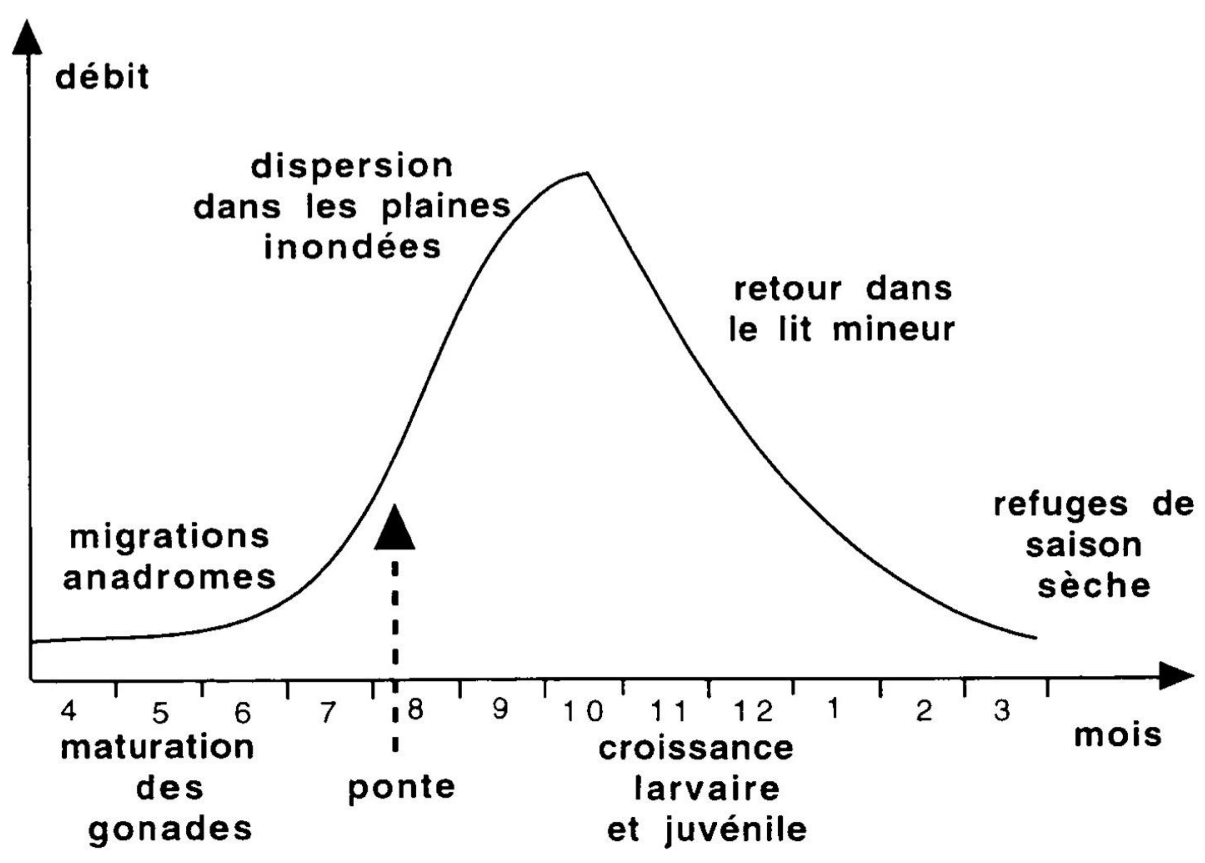

Figure 2 : Dynamique de la crue, ontogenèse, et migrations chez les poissons des rivières tropicales possédant une plaine d'inondation (d'après LOWEMcCONNELL, 1985).

Figure 2 : Seasonal cycle of discharge events, ontogeny, and migrations among riverine fish inhabiting tropical floodplain rivers (from LOWE-McCONNELL, 1985). 


\section{- Un modèle généralisé d'utilisation de l'habitat}

Pour des raisons pratiques et opérationnelles, la nécessité de reconnaître une typologie des habitats a amené certains auteurs à proposer un cadre conceptuel utilisant des caractéristiques physiques et géomorphologiques (FRISSELL et al., 1986). Cette approche hiérarchique peut servir de référence pour l'étude des communautés piscicoles à différentes échelles spatiales et temporelles (BAYLEY et LI, 1992). A grande échelle, ce sont généralement les facteurs liés au climat qui dominent, alors qu'à l'échelle locale les facteurs biotiques comme la prédation ou la compétition peuvent avoir une influence majeure. Mais cette classification a le désavantage de ne pas suffisamment mettre l'accent sur les exigences des poissons en terme d'habitat.

II peut, en effet, être utile dans ce continuum espace-temps d'identifier quelques entités ayant une signification biologique. BAYLEY et LI (1992) ont ouvert cette voie en distinguant quatre grands types d'organisation spatio-temporelle. Selon ces auteurs, le microhabitat correspond à la zone d'activité journalière : alimentation, sélection de meilleures conditions abiotiques, comportement social (grégarisme, territorialité). A l'échelle du mois le domaine d'activité s'étend à la rivière (home range ou domaine vital), alors qu'à l'échelle saisonnière il peut concerner le bassin hydrographique si l'espèce effectue des migrations de grande envergure. Enfin, l'échelle régionale est celle de l'évolution (spéciation) et de la mise en place de faunes sous l'influence d'événements climatiques et géologiques (extinction, colonisation).

Cette typologie proposée par BAYLEY et LI (1992), présente l'intérêt de mettre l'accent sur la relation entre l'utilisation de l'espace et le comportement biologique. Cet effort en vue d'une meilleure définition des habitats basée sur le comportement et les besoins du poisson nécessite cependant d'être poursuivi car il existe un certain flou dans la définition des catégories retenues.

\section{C'est pourquoi je proposerai de reconnaître quatre grands ensembles (Fig. 3) :}

- la zone de stabulation ou zone de repos est l'échelle la plus petite à laquelle un poisson répond à un ensemble plus ou moins complexe de stimuli biotiques et abiotiques. II s'agit avant tout de rechercher une zone où le poisson minimise ses dépenses énergétiques par rapport aux conditions de l'environnement et/ou un abri par rapport aux prédateurs.

- la zone d'activité (home range) à l'intérieur de laquelle les rythmes biologiques et comportementaux sont conditionnés par les cycles nycthéméraux ou lunaires. Le territoire, pour les poissons territoriaux, peut être l'échelle spatiale de référence de cette catégorie. Pour les autres, il s'agit de l'ensemble des zones refuge ou de repos ainsi que des zones où l'espèce va se nourrir, ce qui suppose des migrations de faible amplitude.

Sauf accidents qui les amènent à se déplacer, de nombreuses espèces de poissons territoriaux accomplissent leur cycle biologique dans le contexte spatio-temporel de la zone d'activité. Néanmoins, cette zone d'activité varie lorsque l'environnement aquatique se modifie.

— l'échelle de la niche ontogénique correspond à l'ensemble des milieux dont une espèce a besoin pour accomplir son cycle biologique. Les limites spatiales sont les limites géographiques des différents habitats occupés par les stades de développement, y compris les zones de ponte vers lesquelles l'espèce effectue des migrations parfois importantes lors de la reproduction. Alors que les échelles précédentes concernaient essentiellement l'individu, la niche ontogénique concerne la population dans son ensemble.

- l'échelle régionale, celle de la métapopulation, correspond aux différents bassins hydrographiques dans lesquels l'espèce est présente. Ces bassins sont géographiquement isolés, sauf lors de périodes exceptionnelles à l'échelle géologique.

Les quatre ensembles identifiés ci-dessus correspondent à une complexification croissante au niveau de l'utilisation de l'espace pour les fonctions biologiques (Fig. 4). 


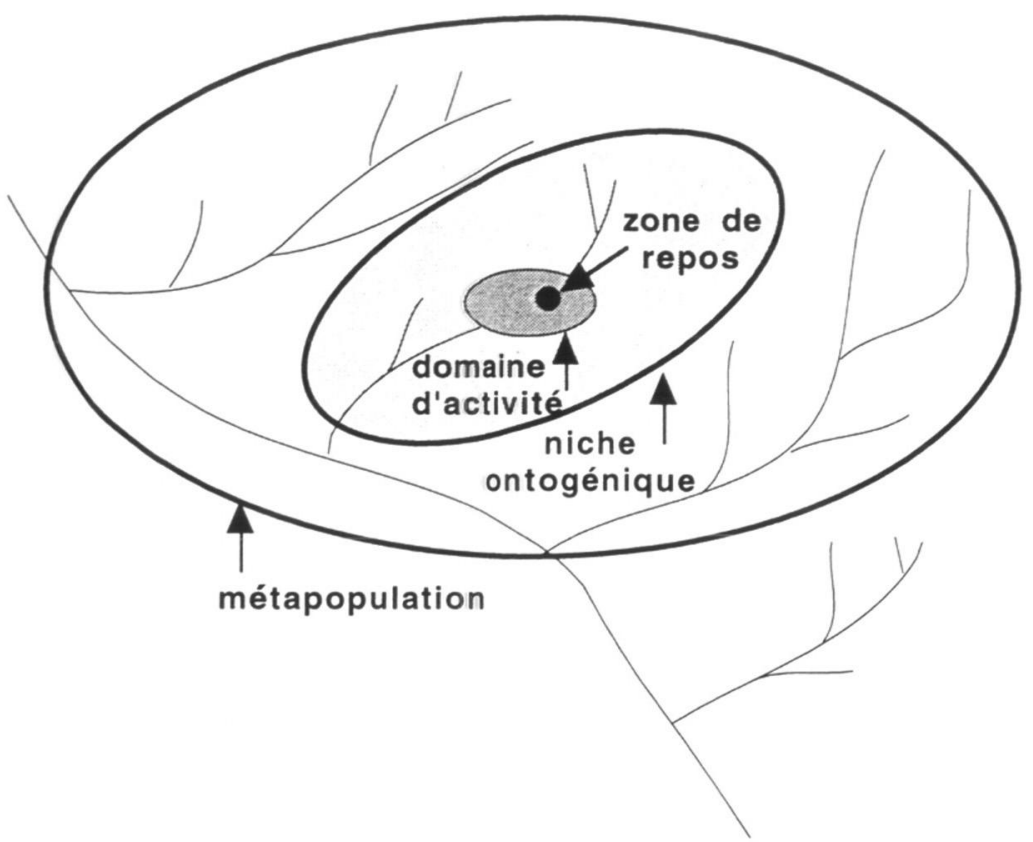

Figure 3 : Les quatre grands ensembles typologiques de l'habitat basés sur les échelles spatiale et temporelle.

Figure 3 : The four main suggested patterns of spatial and temporal models of habitat use.

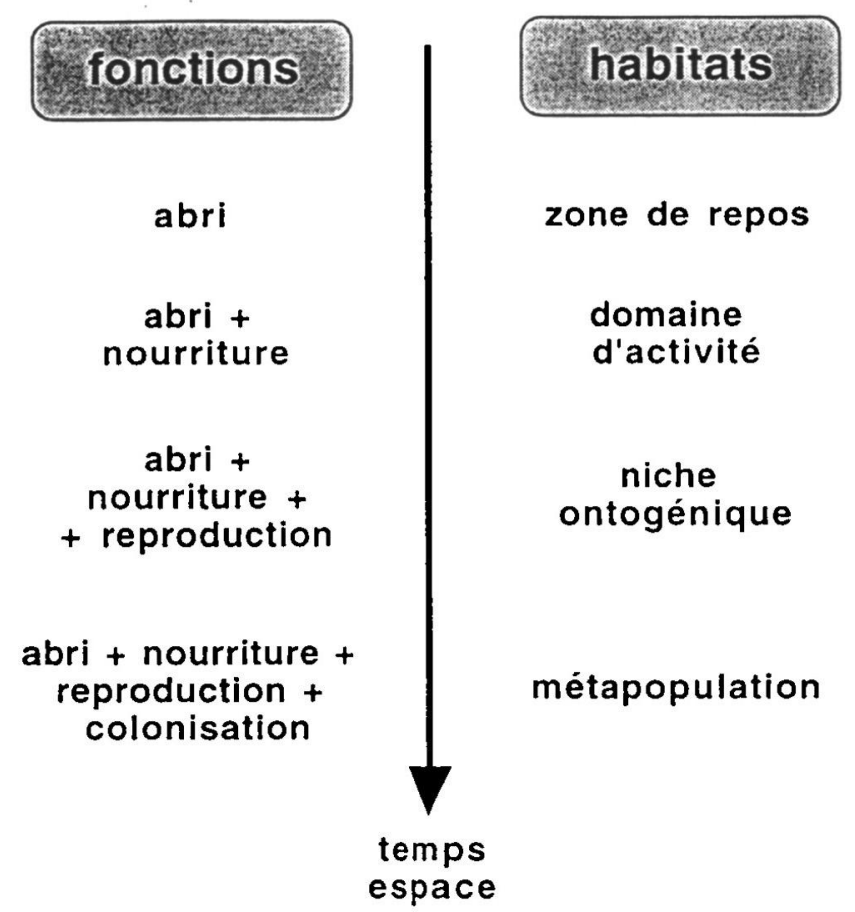

Figure 4 : Relation entre typologie de l'habitat et les fonctions biologiques.

Figure 4 : Correlations between habitat typology and biological functions. 


\section{BIBLIOGRAPHIE}

BALON E.K., 1977. Early ontogeny of Labeotropheus Ahl, 1927 (Mbuna, Cichlidae, Malawi) with a discussion on advanced prospective styles in fish reproduction and development. Environmental Biology of Fishes, 2, 147-176.

BALON E.K., 1985. Early life histories of fishes : new development, ecological and evolutionary perspectives. Developments in Environmental Biology of Fishes 5. W. Junk Publ., Dordrecht.

BALON E.K., 1990. Epigenesis of an epigeneticist : the development of some alternative concepts on the early ontogeny and evolution of fishes. Guelph Ichthyology Reviews, $1,1-42$.

BAYLEY P.B., LI H.W., 1992. Riverine fishes. In Calow P. \& Petts G.E. (eds.), The rivers handbook, volume 1, 251-281, Blackwell Scientific Publications, Oxford.

CÉZILLY F., BRUN B., HAFNER H., 1991. Foraging and fitness. Acta Oecologica, 12, 683-696.

CUSHING D.H., 1982. Climate and fisheries. Academic Press, London.

FRISSELL C.A., LISS W.J., WARREN C.E., HURLEY M.D., 1986. A hierarchical framework for stream habitat classification : viewing streams in a watershed context. Environmental Management, 10, 199-214.

GREENWOOD P.H., 1984. African cichlids and evolutionary theories. In Echelle A.A. \& Kornfield I. (eds.), Evolution of fish species flocks, 141-154, University of Maine at Orono Press, Orono, Maine.

HART P.B.J., 1986. Foraging in teleost fishes. In Pitcher (ed.), The behaviour of teleost fishes, 211-235, Croom Helm, London.

KEENLEYSIDE M.H.A., 1991. Parental care. In Keenleyside M.H.A. (ed.), Cichlid fishes : behaviour, ecology and evolution, 191-208, Chapman \& Hall, Fish and Fisheries Series 2, London.

LAUZANNE L., 1975. Régime alimentaire d'Hydrocyon forskalii (Pisces, Characidae) dans le lac Tchad. Cahiers ORSTOM, série Hydrobiologie, 9, 105-121.

LOWE-McCONNELL R.H., 1985. The biology of the river systems with particular reference to the fishes. In Grove A.T. (ed.), The Niger and its neighbours, 101-140, Balkema.

LOWE-McCONNELL R.H., 1987. Ecological studies in tropical fish communities. Cambridge tropical biology series, Cambridge University Press.

PULLIAM H.R., 1989. Individual behavior and the procurement of essential resources. In Roughgarden J., May R.M. \& Levin S.A. (eds.), Perspectives in ecological theory, 25-38, Princeton University Press, Princeton, New Jersey.

PYKE G.H., 1984. Optimum foraging theory : a critical review. Annual Review of Ecology and Systematics, 15, 523-575.

RIBBINK A.J., 1990. Alternative life-history styles of some African cichlid fishes. Environmental Biology of Fishes, 28, 87-100.

ROSS S.T., 1986. Resource partitioning in fish assemblages : a review of field studies. Copeia, 352-388.

SEMPESKI P., GAUDIN P., 1995. Sélection et utilisation de l'habitat par les jeunes stades de poissons d'eau courante : le modèle ombre commun (Thymallus thymallus, L.). Bull. Fr. Pêche Piscic., 337/338/339, $\mathrm{N}^{\circ}$ spécial Symposium "Le poisson et son habitat". 
WINEMILLER K.O., 1989. Ontogenetic diet shifts and resource partitioning among piscivorous fishes in the Venezuelan ilanos. Env. Biol. Fish, 26, 177-199.

WOOTTON R.J., 1990. Ecology of teleost fishes. Fish and Fisheries series 1, Chapman and Hall, London. 